\title{
A method for detecting preferred scale sizes
}

\author{
Maiken Gustafsson ${ }^{1} \dagger$, Jean-Louis Lemaire ${ }^{2}$ \\ and David Field ${ }^{1}$
}

${ }^{1}$ Department of Physics and Astronomy, University of Aarhus, 8000 Aarhus C, Denmark email: maikeng@phys.au.dk, dfield@phys.au.dk

${ }^{2}$ Observatoire de Paris \& Université de Cergy-Pontoise, LERMA \& UMR 8112 du CNRS, 92195 Meudon, France

\begin{abstract}
Molecular clouds are usually thought to be dominated by turbulence where the structures are inherently self-similar and lack characteristic scale. However self-similarity must break down at scales associated with star formation which imposes a characteristic scale. The turbulence may be driven by energy injection at some larger scale which also imposes characteristic scale. In order to understand the evolution of molecular clouds it is important to identify the departures from self-similarity associated with the scales of self-gravity and the driving of turbulence.

We describe a method based on structure functions for determining whether a region of gas, such as a molecular cloud, is fractal or contains structure with characteristic scale sizes (Gustafsson, Lemaire \& Field 2006). Using artificial data containing structure it is shown that derivatives of higher order structure functions provide a powerful way to detect the presence of characteristic scales should any be present and to estimate the size of such structures. The method is easy to implement and compared with other techniques such as Fourier transform or histogram techniques (Blitz \& Williams), the method appears both more sensitive to characteristic scales and easier to interpret.

The method is applied to observations of hot $\mathrm{H}_{2}$ in the Kleinman-Low nebula, north of the Trapezium stars in the Orion Molecular Cloud, including both brightness and velocity data (Gustafsson, Kristensen, Clénet, et al. 2003). It is found that the density structure, represented by $\mathrm{H}_{2}$ emission brightness in the K-band $(2-2.5 \mu \mathrm{m})$, exhibits mean characteristic sizes of 110 , 550,1700 and $2700 \mathrm{AU}$. The velocity data show the presence of structure at 140,1500 and $3500 \mathrm{AU}$. These scales are respectively disk scales (140 AU) and outflow scales (>1000 AU), the latter being associated with (re-)injection of energy.
\end{abstract}

Keywords. techniques: image processing, ISM: structure, ISM: kinematics and dynamics

\section{References}

Gustafsson, M., Kristensen, L.E., Clénet, Y., Field, D., Lemaire, J.L., Pineau des Forêts, G., Rouan, D., \& Le Coarer, E. 2003, A\&GA 411, 437

Gustafsson, M., Lemaire, J.L. \& Field, D. 2006, A\&A 456, 171

Blitz, L. \& Williams, J.P. 1997, ApJ 488, L145

$\dagger$ Present address: Max-Planck-Institute for Astronomy, Königstuhl 17, 69117 Heidelberg, Germany 